\title{
Los valores profesionales como enfoque educativo ecléctico en el diseño curricular ${ }^{1}$
}

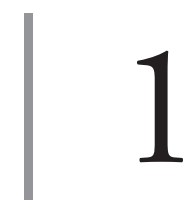

https://doi.org/10.21830/9789585287846.01

Juan Sebastián Alejandro Perilla Granados ${ }^{2}$

El diseño curricular se puede estructurar desde diferentes concepciones, por ello, es común encontrar una diversidad de enfoques para las propuestas de formación. Algunos de estos enfoques centran su interés en los contenidos, otros en las habilidades y otros en las actitudes. Cada uno de estos tres elementos se puede conjugar para consolidar nuevos alcances del diseño curricular, dependiendo de cada uno de los contextos educativos. Es labor del diseñador curricular reconocer los intereses y las necesidades de los actores del proceso de formación para establecer cuál es el enfoque que se exige para realidades específicas.

Las exigencias de un contexto provienen de manera conjunta y dinámica de diferentes actores concretos. Si se tienen en cuenta además los antecedentes, se tiene la posibilidad de comprender a qué punto fundamental se debe enfocar el diseño curricular, evitando recaer sobre temas innecesarios y desconociendo características específicas que tienen la posibilidad de fortalecer el proceso de implementar efectivamente la propuesta de formación que se pretenda realizar. (Perilla, 2018a, pp. 27-28)

Esta conjugación de elementos curriculares, en el marco de procesos de investigación, se puede enmarcar dentro del eclecticismo reflexivo, el cual

1 Este capítulo hace parte de los resultados del proyecto de investigación "Innovación de procesos pedagógicos y curriculares para la enseñanza de las disciplinas" del Grupo de Investigación Invedusa (Investigación Educativa Universidad Sergio Arboleda), registrado con el código COL0160714 de Minciencias. Los puntos de vista y los resultados de este artículo pertenecen al autor y no reflejan necesariamente los de las instituciones participantes.

2 Investigador Senior reconocido por Colciencias. Magíster en Educación. Magíster en Derecho Privado. Especialista en Derecho Comercial y abogado (Universidad de los Andes, Colombia). https:// orcid.org/0000-0001-5283-7601. Contacto: juan.perilla@usa.edu.co 
tiende a establecer propuestas auténticas desde realidades específicas. La autenticidad se configura cuando los diseños curriculares no se centran en repetir las teorías o diseños realizados por otros, sino que, al atender a las características de cada contexto, puede formular una propuesta pertinente para esa realidad. Si bien es una ardua tarea de investigación educativa, se pueden generar propuestas fundamentadas en procesos de validación rigurosos, pues "una propuesta curricular debe ser fruto de un proceso de investigación, es decir, no puede considerarse como algo impuesto o sin un debido fundamento" (Torres, 2010, p. 14).

La validación del enfoque permite que haya confiabilidad al aplicarlo, lo que dota de responsabilidad al proceso de formación. La educación no puede fundamentarse solo en la intuición de quien asume la responsabilidad de enseñar, sino que debe responder a un proceso riguroso, según las exigencias del contexto en conjunto; "la investigación en educación constituye un pilar fundamental para establecer los diferentes diseños curriculares que se pretenden aplicar en las experiencias de formación” (Perilla, 2018b, p. 19). Así, fundamentar procesos de formación en intuiciones puede llevar a que falte confiabilidad y no se alcancen los objetivos pretendidos para cada una de las realidades.

Siendo así, la selección de los enfoques educativos debe responder a un ejercicio de investigación contextual que consustancie las exigencias de diferentes actores; "los procesos de formación no deben contemplar un aprendizaje cualquiera, sino uno que se encamine de forma auténtica a los intereses, necesidades y exigencias de los diferentes actores" (Perilla, 2018c, p. 44). En el marco de este proceso de investigación, desde y para contextos concretos, ha surgido el enfoque de los valores profesionales para la estructuración de currículos. Se trata de una propuesta ecléctica que tiene su fundamentación en diferentes vertientes teóricas y que ha sido adoptada en contextos específicos a partir de trabajos de investigación. Por lo mismo, el presente capítulo busca responder a la siguiente pregunta de investigación: ¡cómo se delimita el proceso de diseño curricular desde el eclecticismo reflexivo para consolidar enfoques educativos como el basado en valores profesionales?

Frente a esta pregunta de investigación se plantea la siguiente hipótesis: para consolidar enfoques educativos como el basado en valores profesionales 
se requiere diseñar el currículo desde los intereses y necesidades de los actores, además, se debe posibilitar la implementación del eclecticismo reflexivo desde y para cada realidad concreta. Así las cosas, el objetivo general de investigación es el siguiente: delimitar el proceso de diseño curricular desde el eclecticismo reflexivo para consolidar enfoques educativos como el basado en valores profesionales. Para alcanzar el objetivo general de investigación se buscará alcanzar los siguientes objetivos educativos a manera de estructura del razonamiento: 1) delimitar al eclecticismo reflexivo como una posibilidad para responder a los intereses y las necesidades desde el diseño curricular y 2) delimitar los enfoques educativos para comprender los valores profesionales como una manera de responder a intereses y necesidades del contexto de formación. Para el desarrollo del presente trabajo se utilizará un enfoque hermenéutico crítico, fundamentado en métodos cualitativos vinculados, de manera preferente, a la revisión documental.

\section{El eclecticismo reflexivo como estrategia de diseño curricular}

La mayoría de las experiencias de formación cuentan con diseños curriculares basados en intereses y necesidades de diferentes fuentes del contexto (Álvarez, 1997; Baeza, 2008; Tyler, 1973). Si bien se espera que se tengan en cuenta los intereses y las necesidades de todas las fuentes para el diseño curricular, esto no sucede en todos los casos, ya que se privilegian unos sobre otros, pues "si la educación es armada como un perfecto discontinuo de concepciones el único perjudicado es, por supuesto, el estudiante” (Toro, 2004, p. 119). Por esto, es recomendable que los diseños curriculares tengan en cuenta intereses y necesidades diversas, para efectos de validar las propuestas de formación y dotarlas de mayor confiabilidad para el logro de sus objetivos.

El diseño curricular es un proceso en el cual confluyen múltiples elementos para generar experiencias de formación, vinculando de forma dinámica objetivos educativos, enfoques, estrategias pedagógicas y de evaluación. En general, los contextos educativos generan procesos encaminados a formar a quienes aprenden y vinculan los mencionados elementos de diferentes maneras. No 
obstante, esta vinculación no siempre responde a procesos de reflexión que evalúen la coherencia de la situación [...] esta falta de reflexión en torno a la selección de elementos curriculares, deja una posibilidad de que el azar determine si se alcanzan o no las intenciones propuestas para cada experiencia formativa. (Perilla, 2018d, p. 41)

En este sentido, entre más fuentes de información se tengan en cuenta para hacer currículos se tiene la posibilidad de asegurar mayor pertinencia al momento de implementar estos (Bernstein, 2001; Stenhouse, 2007; Volante et ál., 2015). De ahí la importancia de saber seleccionar cuáles son las fuentes de información adecuadas y de generar validaciones con ejercicios de investigación rigurosas. En consecuencia, lo primero que se requiere para hacer diseños curriculares es delimitar el contexto en el cual se implementarán (Addine, 2000; Birley, 1972; Zabalza, 2000); el contexto es fundamental puesto que la educación no es un área aislada del conocimiento, sino que pretende impactar una realidad específica. La delimitación del contexto debe tener en cuenta condiciones de tiempo, modo y lugar que permitan proyectar las mejores actividades para los actores del proceso formativo.

La educación cuenta con una serie de contextos que determinan sus características fundamentales y las posibilidades de materializar un enfoque específico. Así, cada contexto cuenta con elementos que lo hacen único y exige que las prácticas de formación tengan un énfasis específico en sus propias dinámicas. A pesar de existir propuestas atractivas desde múltiples perspectivas curriculares, se debe leer primero el contexto para luego ver la pertinencia de las diferentes apuestas pedagógicas que se pretenden alcanzar. (Perilla, 2018e, p. 13)

No obstante, no existe una respuesta exacta sobre cuáles actores se han de tener en cuenta, cómo se deben identificar sus exigencias o cuáles son los instrumentos más pertinentes para tal fin. Depende, en gran medida, de quien diseña el currículo, por lo cual se requiere una notable formación en investigación (Altabach, 2006; Cave, 1971). El diseño curricular no consiste solamente en articular diferentes elementos aparentemente útiles, sino que requiere que se dote de rigor la consolidación de propuestas robustas enmarcadas dentro de objetivos claros y alcanzables, según la realidad en la cual se vayan a aplicar (Cremin, 1975; Cuevas, Pérez y González, 2007). Conocer la realidad desde sus fuentes es el primer paso sugerido para que el diseño curricular pueda alcanzar los fines para los cuales está diseñado. 
Al conocer la realidad del contexto se tendrá un cúmulo variado de información que debe ser evaluada antes de ser incluida en el diseño curricular. Esta información proviene de fuentes diversas que, al ser escuchadas conjuntamente, generan exigencias propias de la realidad que se pretende alcanzar (Delgado, 2011; Delval, 1983). No obstante, la información que se recibe de la realidad debe ser validada de manera reflexiva para determinar cuál es la mejor manera de alcanzar los objetivos de formación. Esa reflexión permite articular elementos de forma auténtica para cada una de las realidades, configurando de esta forma el eclecticismo reflexivo (Posner, 2005). Este último permite que el diseñador del currículo tenga la posibilidad de interpretar su contexto desde diferentes fuentes de información, articulándolas de forma coherente, para dotar de pertinencia cada propuesta de formación específica.

Esto implica que el proceso de diseño curricular deje de ser un ejercicio con respuestas exactas y se constituya en una posibilidad de creación auténtica, que no puede repetirse de manera exacta en contextos diferentes (Gimeno, 2007; Gimeno y Pérez, 1992). Quien diseña el currículo puede tener en cuenta diferentes referentes (Lawton, 1975; Lawton, 1983), pero no será posible apropiar alguno de ellos con aspiración de pureza. Por el contrario, el eclecticismo reflexivo invita a que se tengan en cuenta diferentes fuentes de información, a partir de las cuales se podrán considerar múltiples exigencias y se configurarán, en última instancia, propuestas auténticas desde y para cada una de las realidades que pretenden ser impactadas.

Se hace fundamental que se comprenda que la educación cuenta con actores
concretos que pueden ser vinculados a la realidad para efectos de que las
propuestas pedagógicas no se queden en el discurso, sino que cobren vida
en contextos específicos. La educación necesita procesos vivos que generen
dinamismo y no pretendan ser aplicados de manera forzada al no tener en
cuenta las particularidades concretas de quienes integran el contexto educa-
tivo. (Perilla, 2018f, p. 25 )

Por lo anterior, es fundamental centrarse en aquellas fuentes de las cuales se puede extraer información. Se trata de un ejercicio investigativo que requiere trabajo de campo y revisión bibliográfica, para dotar de sentido a las teorías escritas (Lopes, 2006). No es recomendable configurar un diseńo curricular solamente desde la práctica o desde la teoría, se espera que el 
diseñador curricular pueda conjugar de forma dinámica referentes diversos para asegurar la validación de la propuesta de formación (McKernan, 2008; Zamora y Castejón, 2006). A continuación, se sugieren algunas fuentes de información que se pueden tener en cuenta para construir currículos desde el eclecticismo reflexivo:

1. Quienes aprenden: entendidos generalmente como los estudiantes, aunque no lo son de manera exclusiva. Son todos aquellos sujetos que hacen parte del proceso de formación y tienen la posibilidad de aprender en el mismo (Salas, 2003). Generalmente a ellos se dirigen los objetivos educativos, razón por la cual se hace fundamental que en el diseño curricular se comprendan sus intereses y necesidades, para consolidar propuestas pertinentes para su propia realidad; "en general, los estudiantes tienden a ser los actores más ignorados en el proceso de diseño curricular, pues existe una presunción generalizada de contemplarlos como sujetos vacíos" (Perilla, 2016a, p. 80). Por lo mismo, se ha de permitir que expresen sus exigencias a través de un trabajo de campo, con la utilización de instrumentos de recolección de información que den cuenta de aquello que requieren estos actores (Schwab, 1969).

2. Quienes enseñan: por lo general son los profesores, pero al igual que los anteriores pueden variar. Dependerá de quiénes son aquellos que están en la posibilidad de asegurar aprendizajes en otros. Por regla general, son los encargados de materializar las apuestas del diseñador curricular y, en consecuencia, se deben tener en cuenta al momento de plantear los procesos formativos; "toda investigación y todo desarrollo bien fundamentado del currículo [...] descansa, por lo tanto, en el trabajo de los profesores" (Stenhouse, 1998, p. 54). Se necesita saber qué requieren estos sujetos antes, durante y después del proceso de formación, para que la propuesta sea pertinente. Como se trata de sujetos que se encuentran en la cotidianidad del contexto, en la mayoría de los casos también se requiere identificar sus exigencias desde instrumentos de recolección de información de campo (Tovar y Sarmiento, 2011). 
3. El medio educativo: se trata de una de las fuentes de información más ambigua de todas, pero que, por lo mismo, se hace fundamental delimitar. El medio educativo está conformado por todos aquellos actores que rodean e inciden de manera significativa en quienes enseñan y quienes aprenden; "su delimitación puede tener un alcance estratégico, pues contribuye junto con los demás actores a la definición del currículo" (Perilla, 2016a, p. 82). Pueden estar en esta categoría actores gubernamentales (Ministerio de Educación, Secretarías de Educación o entidades análogas), directivos institucionales (rectores, coordinadores, etc.) e incluso los padres de familia; "la escuela es la comunidad organizada básica en educación y es a este nivel donde hay que tratar los problemas y las posibilidades de la innovación del currículo” (Stenhouse, 1998, p. 222). En cualquier caso, quien diseña el currículo debe tenerlos en cuenta para efectos de determinar sus exigencias a través de recolección de información en el campo o a través de revisión documental.

4. Expertos disciplinares: aquellos que plantean los referentes teóricos de aquello que se pretende enseñar; "el experto disciplinar puede contribuir al diseño curricular en la superación del debate acerca de cuáles son los contenidos que deben ser o no incluidos" (Perilla, 2016a, p. 85). Los procesos de formación cuentan con conocimientos específicos que deben estar fundamentados, caso en el cual estos expertos deben ser delimitados a través de estados del arte. Por lo mismo, son aquellos actores que han generado publicaciones especializadas en áreas objeto de enseñanza y aprendizaje, los cuales deben analizarse en relación con todos aquellos que sea posible (Taba, 1962). Se trata de una revisión documental de publicaciones de investigación que lleven a fundamentar el proceso formativo (Sarmiento y Tovar, 2007).

5. Expertos curriculares: son aquellos autores que fundamentan las prácticas pedagógicas al enseñar y al aprender. Se hace fundamental que los currículos no se centren solamente en los conocimientos que deben ser aprendidos, sino que se complementen con propuestas 
sobre cómo enseñar, aprender y evaluar (Timmerman, Strickland y Carstensen, 2008); "los expertos curriculares contribuyen a determinar cuál debe ser la orientación de los diferentes procesos de formación según el contexto del cual se trate" (Perilla, 2016a, p. 87). Se trata de una de las principales falencias en los diseños curriculares, la cual puede ser superada con la consolidación de estados del arte especializados en formar desde y para realidades análogas a aquella que se desea impactar.

La anterior no es una lista taxativa, sino unos mínimos recomendables para el diseño curricular. Lo que se espera es que de cada uno de los actores se obtengan sus respectivos intereses y necesidades, de tal manera que sea posible extraer de ellos determinadas exigencias de formación. Estas exigencias plantearán diferentes retos que deben ser tenidos en cuenta por aquel que diseña el currículo, para construir la propuesta que deberá ser implementada en cada contexto (Anderson, 2002; Barreto, 2013). Al conjugar los diferentes actores se requiere crear una propuesta auténtica, que por la diversidad de sus fuentes difícilmente podrá ser reproducida con exactitud en otros contextos específicos (Dillon, 2009; Herman, Webb y Zúñiga, 2007). En esa diversidad se encuentra la riqueza del eclecticismo reflexivo, dado que se generan propuestas de formación pertinentes a través de la vinculación de las exigencias que plantea el contexto de formación.

De esta manera, el eclecticismo reflexivo se constituye en una propuesta de diseño curricular que, además de trascender la formalidad de las propuestas teóricas o los lineamientos del campo, atiende a diferentes referentes para consolidar propuestas de formación auténtica, validada y confiable. Se superan así las dinámicas de repetir modelos o propuestas sobre cómo enseñar creadas en contextos ajenos-, para, en cambio, dotar de sentido de pertinencia aquello que se quiere lograr con sujetos específicos. Pero no se trata solamente de mezclar por mezclar, sino que se requiere construir propuestas diversas desde la reflexión rigurosa que otorga la investigación.

Una de las manifestaciones más notables del eclecticismo reflexivo se encuentra en los enfoques educativos que se aplican en diferentes contextos. Se han propuesto enfoques desde elementos como los contenidos, las habilidades 
y las actitudes. No obstante, la implementación de los mismos ha llevado a configurar propuestas eclécticas que en sí mismas son innovaciones pedagógicas. Una de estas propuestas eclécticas está conformada por enfoques como el fundamentado en valores profesionales, el cual se procederá a delimitar como resultado de la conjugación de diferentes enfoques a partir del eclecticismo reflexivo.

\section{Los valores profesionales como enfoque educativo ecléctico}

En el marco de las dinámicas del eclecticismo reflexivo, tendientes a considerar diferentes fuentes de información para el diseño curricular, uno de los elementos que más derivaciones auténticas ha tenido es el de los enfoques educativos; "cada diseño curricular cuenta con un enfoque educativo que determina la forma de interpretar y articular los diferentes elementos alrededor de una experiencia de formación" (Perilla, 2018g, p. 44). Los enfoques educativos generan lineamientos sobre cómo formar a los actores y gestionar las propuestas curriculares desde su implementación (Posner, 2005). Al mismo tiempo, diferencian las prácticas educativas entre contextos y aseguran autenticidad en su creación.

El enfoque educativo es aquella impronta que busca justificar los elementos educativos y determinar cuáles son aplicables a cada escenario en particular. Este enfoque se establece a partir de los objetivos educativos propuestos para cada proceso, de tal suerte que se asegure alineación curricular entre lo que se quiere y la forma de alcanzarlo. (Perilla, 2016b, p. 27)

Se han generado tantos enfoques educativos como contextos, aunque no todos están plenamente sistematizados. En ocasiones, son prácticas recurrentes que carecen de procesos de investigación, pero esto no significa que no se presenten permanentemente en los procesos de formación. La tarea del diseñador del currículo es que su propuesta de formación no se quede solamente en una anécdota institucional o del contexto, sino que pueda tener un soporte de sistematización rigurosa. Esto toma especial relevancia si se considera que cada contexto tiene su propio enfoque educativo y se hace fundamental promover su interpretación desde realidades específicas. 
Los contextos educativos tienen características propias que determinan un sello específico al momento de materializar los procesos de formación. Así, se pueden compartir elementos curriculares y pedagógicos entre diferentes contextos, pero cada experiencia de enseńanza y aprendizaje cuenta con particularidades que la hacen única respecto de otras. (Perilla, 2018h, p. 49)

Los diversos enfoques que existen se pueden agrupar a partir de los diferentes alcances de su conocimiento, a saber: los contenidos, las habilidades y las actitudes. Estos alcances del conocimiento han sido catalogados como una serie de saberes: los contenidos hacen referencia al saber conocer, las habilidades al saber hacer y las actitudes al saber ser. Cada uno de estos elementos dependerá del objetivo que se persiga con el diseño curricular, el cual resulta de la exigencia que proviene de intereses y necesidades particulares. En ocasiones la exigencia del contexto se centra en el aprendizaje de contenidos, por lo cual los enfoques educativos tenderán a ser tradicionales. ${ }^{3}$ Por otra parte, cuando la exigencia se centra en el aprendizaje de habilidades se está en presencia de enfoques experienciales. ${ }^{4} \mathrm{Y}$ finalmente, los que buscan el desarrollo de actitudes tenderán a ser actitudinales. ${ }^{5}$ Cada uno de estos enfoques será o no pertinente según las características propias de cada contexto. La conjugación entre ellos ha generado diferentes posibilidades para articular las prácticas educativas.

Es difícil aplicar un enfoque educativo puro en un contexto particular, pues los diseños curriculares que tienen en cuenta las necesidades e intereses de

3 "El enfoque tradicional se centra, de manera principal, en los contenidos y surge bajo la necesidad de conservar información del legado cultural. Así, este enfoque surge en aquellas tribus antiguas que no tenían la posibilidad de escribir y la preservación de sus tradiciones se hacía a través de la tradición oral. En este sentido, los grupos de personas se reunían en torno a la persona que mayor experiencia tenía en la tribu, quien les transmitía información específica de su cultura y sus prácticas sociales. Los demás memorizaban lo transmitido, de tal manera que se preservaba la información de su propia comunidad" (Perilla, 2018i, p. 22).

4 "El enfoque experiencial no se centra en los contenidos, sino en el desarrollo de habilidades en los estudiantes. Se enmarca en una lógica de saber hacer, en la que la educación técnica cobra una importancia especial. Esta formación no se pregunta sobre el porqué de determinada acción, sino que la misma se aprende desde el cómo con procesos de repetición constante” (Perilla, 2018i, p. 23).

5 "El enfoque actitudinal no se centra en contenidos ni en habilidades, sino que, al seguir su nombre, este se centra en actitudes. Así, para este enfoque lo fundamental es que el estudiante se sienta pleno desde sus características personales. La preocupación de quien diseña el currículo consiste en asegurar que los estudiantes tengan la posibilidad de sentirse reconocidos desde su diversidad, motivados a aprender, felices con ellos mismos y vean en el proceso de formación una significativa oportunidad para fortalecer su formación personal" (Perilla, 2018i, p. 25). 
los intervinientes requerirán enfoques que sean producto del eclecticismo reflexivo. (Posner, 2005)

El eclecticismo reflexivo exige que el diseñador del currículo realice la formulación de los diferentes elementos curriculares, teniendo en cuenta múltiples referentes teóricos. (Perilla, 2017, p. 41)

En este sentido, "se requiere que a través de un proceso de revisión de las propuestas teóricas y de las características cotidianas de los contextos, se generen las condiciones específicas para la implementación de los diferentes enfoques" (Perilla, 2018j, p. 58). A manera de ejemplo, se puede considerar que al conjugar los contenidos con las habilidades se podrán alcanzar enfoques de estructura de las disciplinas. Por su parte, la conjugación entre habilidades y actitudes puede llevar a configurar un enfoque conductual. Finalmente, la mezcla entre actitudes y contenidos puede derivar en un enfoque cognitivo. $\mathrm{Al}$ conjugar todos los elementos anteriores se puede estar en presencia de un enfoque de aprendizaje basado en competencias e incluso uno constructivista. Pero, en cualquier caso, se tenderá a generar derivaciones de cada uno de ellos según la realidad de la cual se trate. Esta relación de enfoques se puede hacer evidente en el diagrama que presenta la figura 1:

Figura 1. Relación de contenidos, habilidades y actitudes con el enfoque del Aprendizaje. Basado en Competencias (ABC)

(1) Enfoque tradicional.

(2) Enfoque experiencial.

(3) Enfoque actitudinal.

(4) Enfoque estructura de las disciplinas.

(5) Enfoque conductual.

(6) Enfoque cognitivo.

ACTITUDES

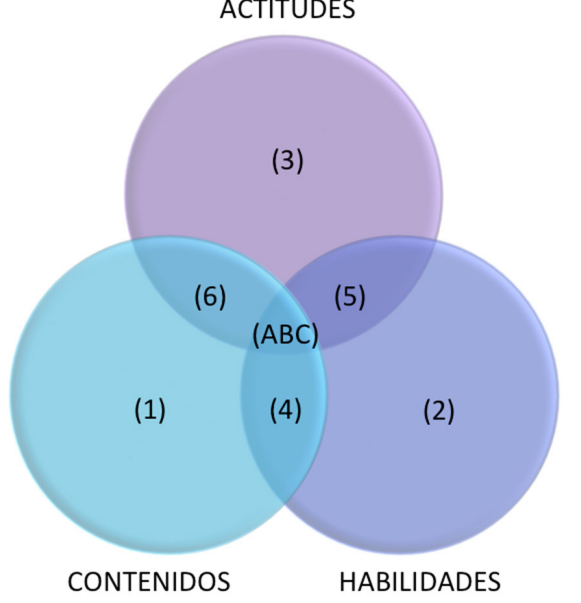

Nota. Tomado de "Los enfoques educativos desde el diseño curricular", por J. Perilla, 2018i, Universidad Sergio Arboleda y Alcaldía Mayor de Bogotá. 
Algunos contextos específicos han centrado sus preocupaciones de formación en los valores profesionales, donde el objetivo educativo del diseño curricular pretende desarrollarlos. En este caso, ninguno de los enfoques antes sugeridos puede responder de manera exacta al logro del objetivo propuesto. Esto se justifica al considerar que los valores profesionales no se limitan solamente a un tema de contenidos, de habilidades o de actitudes. Probablemente, se requiere la adopción de diferentes elementos para alcanzar lo que se pretende con este proceso formativo, lo que exige una reflexión propia del eclecticismo reflexivo. Esta reflexión podría sugerir, en un primer momento, que los valores se centran en una actitud, por lo cual se podrá ubicar en el enfoque actitudinal de manera preferente. No obstante, al no ser valores abstractos sino, en cambio, tener un alcance profesional, se requerirá tanto de habilidades para poner en práctica determinados desempeńos como de conocimiento en contenidos propios de la disciplina para fundamentar el proceso formativo.

La conjugación de elementos hace que para el desarrollo de valores profesionales se requiera materializar un enfoque ecléctico marcadamente actitudinal, con elementos puntuales de las habilidades y de los contenidos. Esto implica que el enfoque educativo, fundado en valores profesionales, pueda ser, en sí mismo, una propuesta auténtica que toma elementos de varios enfoques, para llegar a una práctica educativa específica. No obstante, el contenido concreto de los valores dependerá del contexto en el cual se pretenda desarrollarlos. Considerando que los diseños curriculares no pueden limitarse a ser una construcción desde la teoría, el enfoque educativo deberá complementarse con información de campo recopilada directamente de los actores.

La educación debe trascender las paredes de la escuela para que, a partir de la solución de problemas concretos, impacte a los actores del proceso de formación y de esta manera sea posible que repliquen los avances formativos en diferentes ámbitos del conglomerado social. Es una propuesta que se debe seguir fortaleciendo desde diferentes enfoques, de tal como que se generen elementos eclécticos cada vez que se aplique y que siempre propenda a asegurar que la educación sea una efectiva herramienta de transformación social. (Perilla, 2018k, p. 33)

Por lo mismo, el alcance del enfoque educativo fundamentado en valores profesionales podrá tener un alcance diverso según el contexto en el cual se 
aplique. No sería dable definirlo desde una perspectiva teórica o de campo única, sino que han de tenerse en cuenta diferentes fuentes de información para aportar de manera significativa a la comprensión real de su alcance. Esto implica que el alcance de los valores podrá ser indeterminado hasta que se delimite específicamente el contexto específico que se quiere impactar. Es ahí donde cada uno de los actores debe confluir para darle un alcance pertinente a lo que se entiende por valores profesionales; será esa la tarea que asumirá esta obra a partir de los capítulos posteriores. He ahí la enorme posibilidad de asegurar que el eclecticismo reflexivo dote de sentido a enfoques desde y para realidades específicas, como lo es el caso de los valores profesionales.

Los procesos de formación requieren diseńos curriculares desde las exigencias de cada uno de los contextos para los cuales se dirigen, por lo cual es fundamental desarrollar investigaciones que conjuguen información recolectada en campo y consultada en fuentes bibliográficas. Esta información requiere de la vinculación de diferentes actores específicos, a saber: quienes aprenden, quienes enseñan, el medio educativo, los expertos disciplinares y los expertos curriculares. Cada uno de ellos puede aportar sus intereses y necesidades, de tal manera que, en conjunto, se generen diseños curriculares pertinentes para la realidad que pretenden impactar.

Esto implica que quien diseña el currículo debe desarrollar procesos de investigación permanentes, con instrumentos de recolección de información de campo o revisiones bibliográficas tendientes a construir estados del arte. De este modo, cada uno de los contextos educativos genera aportes que hacen diseños curriculares auténticos, es decir, con características que los diferencian de otras realidades, dadas las particularidades específicas con las que cuentan. Es labor del diseñador curricular identificar todos estos elementos para que desde la reflexión sea posible construir propuestas de formación eclécticas.

En conformidad con lo anterior, el eclecticismo reflexivo se da cuando al diseñar una propuesta curricular de formación se toman diferentes elementos para conjugarlos según las exigencias de un contexto concreto. El contexto exige, entonces, que se conjuguen referentes desde la teoría y la práctica, como ejercicios de validación que generan confiabilidad al momento de implementar una propuesta curricular. Tal es el caso de los enfoques educativos, los cuales 
se han estructurado desde el análisis de diferentes exigencias materializadas en objetivos educativos. Algunas de estas exigencias se estructuran desde los contenidos, otras desde las habilidades y otras desde las actitudes.

Aquellos diseños curriculares cuyas exigencias se fundamentan en los contenidos tenderán a ser catalogados como tradicionales, los que giran en torno a habilidades como experienciales y los de actitudes como actitudinales. De la conjugación de todos estos enfoques pueden surgir otros, como el de estructura de las disciplinas, el conductual, el cognitivo, el de aprendizaje basado en competencias e incluso el constructivista. Cada uno de los enfoques tendrá características relevantes en su contexto, por lo cual es fundamental generar sistematizaciones de sus prácticas, lo que permite que no se trate de procedimientos aislados, sino que aporten a la consecución de objetivos concretos.

Ahora bien, en el marco de esos enfoques surge un interés especial por parte de algunos contextos referentes a los valores profesionales. Cuando este interés se materializa como exigencia de un contexto, el objetivo educativo exige la configuración de un enfoque desde el eclecticismo reflexivo. Así, desde una perspectiva teórica, se tendrá que esta exigencia requiere de manera especial de determinadas actitudes, las cuales han de ser complementadas, en parte, por ciertas habilidades y contenidos. Las actitudes aseguran una manera de ser, al tiempo que las habilidades promueven desempeños específicos y los contenidos saberes disciplinares concretos.

No obstante, para la construcción real del enfoque de valores profesionales se ha de interpretar este desde la realidad concreta de cada contexto. Por lo mismo, se tiene un alcance indeterminado del enfoque, hasta que se proceda a complementarlo con intereses y necesidades de los actores de la realidad que se pretende impactar. Es tarea del diseñador del currículo hacer un ejercicio de investigación fundado en la reflexión, el cual permitirá conjugar diferentes elementos para llegar a una propuesta de formación ecléctica. Se avala de esta manera la hipótesis del presente capítulo, al tiempo que la respuesta a su pregunta y el alcance de sus objetivos permitirán delimitar en los capítulos siguientes los valores profesionales como un enfoque ecléctico desde y para realidades específicas. 


\section{Referencias}

Addine, F. (2000). Diseño curricular. Instituto Superior Pedagógico Enrique José Varona.

Altabach, P. (2006). Internacional Higher Education: Reflections on Policy and Practice. Boston College CIHE.

Álvarez, R.M. (1997). Hacia un curriculo integral y contextualizado. Ediciones Academia.

Anderson, I. (2002). Curricular alignment: a re-examination. Theory into Practice, 41(4), 255-260.

Baeza, J. (2008). El diálogo cultural de la escuela y en la escuela. Estudios Pedagógicos, 34(2), 193-206.

Barreto, N. (2013). El discurso didáctico en la transformación del currículum para la formación de profesores: Espacio que reclama definición. Investigación y Postgrado, 28(2), 125-153.

Bernstein, B. (2001). La estructura del discurso pedagógico. Ediciones Morata.

Birley, D. (1972). Planning and education. Routledge and Kegan Paul.

Cave, R. (1971). An introduction to curriculum development. Ward Look Educational.

Cremin, L. A. (1975). Curriculum-making in the United States. En W. Pinar (ed.), Curriculum theorizing: The Reconceptualists. Berkeley: McCutchan.

Cuevas, L. (2007). El Programa de Desarrollo Curricular. La experiencia en el diseño y desarrollo de planes de estudio por competencias profesionales en el Centro Universitario de Ciencias de la Salud. Revista de Educación y Desarrollo, 6, 63-72.

Delgado, C. (2011). Transdisciplina, currícula universitaria e investigación. En: J. García, J. Betancourt y F. Martínez (coord.), Transdisciplina y desarrollo humano (pp. 132-151). Dirección de Calidad y Enseñanza en Salud de la Secretaría de Salud del Estado de Tabasco.

Delval, J. (1983). Creer y pensar: la construcción del conocimiento en la escuela. Laia.

Dillon, J. (2009). On scientific literacy and curriculum reform. International Journal of Environmental \& Science Education, 4(3), 201-213.

Gimeno, J. (2007). El currículum: una reflexión sobre la práctica. Editorial Morata.

Gimeno, J. y Pérez, A. (1992). Comprender y transformar la enseñanza. Ediciones Morata.

Herman, J., Webb, N. y Zuñiga, S. (2007). Measurement issues in the alignment of standards and assessments. Applied Measurement in Education, 20(1), 101-126.

Lawton, D. (1975). Class, culture and the curriculum. Routledge and Kegan Paul.

Lawton, D. (1983). Curriculum studies and curriculum planning. Hodder and Stoughton.

Lopes, A.C. (2006). Relaçóes macro/micro na pesquisa em currículo. Cadernos de Pesquisa, 36(129), 619-635.

McKernan, J. (2008). Investigación-acción y curriculum: métodos y recursos para profesionales reflexivos. Ediciones Morata.

Perilla Granados, J. S. A. (2016a). La cultura de la investigación como fundamento de diseño curricular. En M. H. Arana Ercilla y V. H. Ibarra Argoty (comps.), Cultura de la investigación y gestión educativa: experiencia desde tres universidades colombianas (pp. 69-91). Universidad Sergio Arboleda y Escuela Militar de Cadetes "General José María Córdova”. 
Perilla, J. (2016b). Alineación iusteórica de los enfoques educativos para las facultades de derehco. En J. Perilla (coord.), Debates Educativos Contemporáneos en Contexto (pp. 15-42). Universidad Sergio Arboleda.

Perilla, J. (2017). Constructivismo antiformalista: conceptualización pedagógica y materialización jurídica. Universidad Sergio Arboleda. doi: 10.22518/9789588987187

Perilla, J. (2018a). Estrategias de diseño curricular desde antecedentes y exigencias contextuales. En J. Perilla (comp.), Diseño curricular y transformación de contextos educativos desde experiencias concretas (pp. 13-40). Universidad Sergio Arboleda y Alcaldía Mayor de Bogotá.

Perilla, J. (2018b). Hacia una investigación antiformalista. En J. Perilla (comp.), Experiencias de innovación educativa desde investigaciones antiformalistas (pp. 19-42). Universidad Sergio Arboleda y Alcaldía Mayor de Bogotá.

Perilla, J. (2018c). Los niveles de conocimiento del aprendizaje y su relación con los procesos de investigación en contextos educativos. En J. Perilla (comp.), Experiencias de innovación educativa desde investigaciones antiformalistas (pp. 43-70). Universidad Sergio Arboleda y Alcaldía Mayor de Bogotá.

Perilla, J. (2018d). La alienación curricular como una estrategia para el fortalecimiento de los contextos educativos. En J. Perilla (comp.), Diseño curricular y transformación de contextos educativos desde experiencias concretas (pp. 41-68). Universidad Sergio Arboleda y Alcaldía Mayor de Bogotá.

Perilla, J. (2018e). Hacia un constructivismo ecléctico desde el misreading. En J. Perilla (Comp.), Constructivismo ecléctico desde la reflexión curricular (pp. 13-42). Universidad Sergio Arboleda y Alcaldía Mayor de Bogotá.

Perilla, J. (2018f). La transformación social desde roles dinámicos de los actores de cada contexto educativo. En J. Perilla (comp.), La transformación social desde el protagonismo del estudiante y la convivencia escolar (pp. 17-50). Universidad Sergio Arboleda y Alcaldía Mayor de Bogotá.

Perilla, J. (2018g). Estrategias pedagógicas para vincular los preconceptos desde el constructivismo. En J. Perilla (comp.), Constructivismo ecléctico desde la reflexión curricular (pp. 43-74). Universidad Sergio Arboleda y Alcaldía Mayor de Bogotá.

Perilla, J. (2018h). Consolidación dinámica de enfoques educativos centrados en el estudiante para la transformación social. En J. Perilla (comp.), La transformación social desde el protagonismo del estudiante y la convivencia escolar (pp. 51-72). Universidad Sergio Arboleda y Alcaldía Mayor de Bogotá.

Perilla, J. (2018i). Los enfoques educativos desde el diseño curricular. En J. Perilla (comp.), Aprendizaje basado en competencias: un enfoque educativo ecléctico desde y para cada contexto (pp. 19-42). Universidad Sergio Arboleda y Alcaldía Mayor de Bogotá.

Perilla, J. (2018j). Las competencias como un enfoque educativo para el diseño curricular desde el eclecticismo reflexivo. En J. Perilla (comp.), Aprendizaje basado en competencias: un enfoque educativo ecléctico desde y para cada contexto (pp. 43-68). Universidad Sergio Arboleda y Alcaldía Mayor de Bogotá.

Perilla, J. (2018k). La investigación en educación y su enfoque como herramienta de transformación social. En M. H. Arana Ercilla y V. H. Ibarra Argoty (Comps.), Ideas y experiencias 
pedagógicas: investigación formativa y la tutoria de trabajo de grado en la educación superior (pp. 11-35). Escuela Militar de Cadetes General José María Córdova.

Posner, R. (2005). Análisis del currículo. McGraw-Hill.

Salas, R. (2003). La identificación de necesidades de aprendizaje. Educación Médica Superior, 17(1), 25-38.

Sarmiento, P. y Tovar, M. C. (2007). El análisis documental en el diseño curricular: un desafío para los docentes. Colombia Médica, 38(4), 54-63.

Schwab, J. (1970). The practical: a language for curriculum. National Education Association.

Stenhouse, L. (1998). Investigación y desarrollo del curriculum. Morata.

Stenhouse, L. (2007). La investigación como base de la enseñanza (6ta ed.). Morata.

Taba, H. (1962). Curriculum development, theory and practice. Harcourt Brace and World Inc.

Timmerman, B., Strickland, D. y Carstensen, S. (2008). Curricular reform and inquiry teaching in biology: where are our efforts most fruitfully invested? Integrative and Comparative Biology, 48(2), 226-240.

Toro, J. (2004). La autonomía, el propósito de la educación. Revista de Estudios Sociales, (19), 119-124. doi: http://doi.org/10.7440/res19.2004.10

Torres, G. (2010). Currículo y evaluación. El Currículo, 15.

Tovar, M. C. y Sarmiento, P. (2011). El diseño curricular, una responsabilidad compartida. Colombia Médica, 42(4), 508-517.

Tyler, R. (1973). Principios básicos del currículo. Editorial Troquel.

Volante, P., Bogolasky, F., Derby, F. y Gutiérrez, G. (2015). Hacia una teoría de acción en gestión curricular: Estudio de caso de enseñanza secundaria en matemática. Psicoperspectivas, 14(2), 96-108.

Zabalza, M. (2000). Diseño y desarrollo curricular (5ta. ed.). Narcea.

Zamora, M. y Castejón, H. (2006). Procesos de cambio y transformación: competencias del perfil profesional del orientador. Paradigma, 27(1), 349-36 